\title{
Ropes on a line embedded in a Grassmannian variety
}

\author{
Edoardo BALLICO, Roberto NOTARI \\ and Maria Luisa SPREAFICO \\ Dipartimento di Matematica, \\ Dipartimento di Matematica, \\ Università di Trento, \\ I-38050 Povo (Tn), Italy \\ Politecnico di Torino, \\ I-10129 Torino, Italy \\ ballico@science.unitn.it \\ roberto.notari@polito.it \\ Dipartimento di Matematica, \\ Politecnico di Torino, \\ I-10129 Torino, Italy \\ maria.spreafico@polito.it
}

Recibido: 21 de Octubre de 2002

Aceptado: 13 de Octubre de 2003

\begin{abstract}
Let $L$ be a line contained in a Grassmannian variety $G$. A $d$-rope $C \subset G$ supported on $L$ is a locally Cohen-Macaulay curve of degree $d$ with $C_{\text {red }}=L$ and $\left(\mathcal{I}_{L, G}\right)^{2} \subset \mathcal{I}_{C, G}$. We characterize the $d$-ropes $C$ supported on $L$ and embedded in $G$. In some cases we describe also the vector bundles on such a rope $C$. Finally, we describe the parameter spaces for ropes embedded in $G$.
\end{abstract}

Key words: multiple line, Grassmannian variety, normal sheaf, embeddings.

2000 Mathematics Subject Classification: Primary 14C05, 14M15; Secondary 14H99

\section{Introduction}

Given a smooth curve $L$, a multiple structure $C$ supported on $L$ is a curve with $C_{\text {red }}=L$, where a curve is a locally Cohen-Macaulay scheme of pure dimension 1 . Particular multiple structures are the so-called $d$-ropes, where a $d$-rope is a degree $d \cdot \operatorname{deg} L$ curve whose ideal sheaf satisfies $\left(\mathcal{I}_{L}\right)^{2} \subset \mathcal{I}_{C} \subset \mathcal{I}_{L}$, i.e. its relative ideal sheaf $\mathcal{I}=\mathcal{I}_{L, C}$ satisfies $\mathcal{I}^{2}=0$. 
Geometrically the curve $C$ is contained between $L$ and its first infinitesimal neighborhood. It is easy to see that a $d$-rope $C$ of degree $d \cdot \operatorname{deg} L$ corresponds to a rank $d-1$ subbundle $E$ of the normal bundle $\mathcal{N}_{L}$ of $L$ via the exact sequence

$$
0 \rightarrow E^{*}=\mathcal{I}_{L, C} \longrightarrow \mathcal{O}_{C} \longrightarrow \mathcal{O}_{L} \rightarrow 0 .
$$

We say that $C$ is a rope if it is a $d$-rope for some $d$.

The definition of rope was given in [5], while 2-ropes (called ribbons) and $d$-ropes embedded in the projective space were studied in [1] and [9], respectively.

If the support $L$ is a line and the rope $C$ is embedded in $\mathbb{P}^{n}$, the Hilbert function, the homogeneous ideal, the Hartshorne-Rao module, their biliaison classes and their Hilbert schemes were studied in [10] and [11]. Moreover, curves $C$ contained between two infinitesimal neighborhoods of a line $L$, embedded in $\mathbb{P}^{n}$, were studied in [2].

In this work we want to study ropes $C$ supported on a line $L$, both embedded in a Grassmannian variety $G$. This fact seems quite natural because the Grassmannian varieties are a generalization of the projective spaces $\mathbb{P}^{n}$.

The plan of the paper is the following.

In Section 2 we give a characterization of the bundles

$$
E^{*}=\mathcal{I}_{L, C}=\oplus_{i=1}^{d-1} \mathcal{O}_{L}\left(\alpha_{i}-1\right)
$$

(see the previous exact sequence (1)) which define $d$-rope $C$ embedded in the Grassmannian variety $G$. Furthermore, we deduce some numerical invariants of such embedded ropes (for example we easily prove that $g_{C}=-\sum_{i=1}^{d-1} \alpha_{i}$, where $g_{C}$ is the arithmetic genus of $C$ ).

In Section 3 we describe the vector bundles $A$ on a $d$-rope $C$ embedded in $G$, supported on a line $L$, which satisfy the condition $A_{\mid L}$ is rigid.

Recalling that every Grassmannian variety $G=G_{r, n}$ can be embedded via Plücker morphism in the projective space $\mathbb{P}^{N}$ with $N=\left(\begin{array}{l}n \\ r\end{array}\right)-1$, in Section 4 we show that for almost every $d$-rope $C$ embedded in $G \subset \mathbb{P}^{N}$, there exists a $d^{\prime}$-rope $C^{\prime} \subset \mathbb{P}^{N}$ such that $C$ is the scheme-theoretical intersection of $C^{\prime}$ with $G$.

Finally, in the last section we study the parameter space for the $d$-ropes embedded in $G$. Whenever we choose two suitable parameter spaces we describe a flat family of $d$-ropes embedded in the same Grassmannian variety $G$ such that the general element belongs to one of the two parameter spaces and the special one belongs to the other one.

\section{Characterizations of ropes supported on a line, embedded in the Grassmannian variety}

In this section, we consider a line $L$ contained in a Grassmannian variety $G$ and we give a characterization of a $d$-rope $C$ supported on a line $\mathrm{L}$ both embedded in $G$.

Throughout this paper we work over an algebraically closed field $K$ of any characteristic and we'll use the following notation. Let $G:=G_{r, n}$ be the set of $r$-dimensional 
linear subspaces of the vector space $K^{n}$. Of course each $r$-dimensional linear subspace of $K^{n}$ can be view as a $(r-1)$-plane in the corresponding projective space $\mathbb{P}^{n-1}$. We have that $\operatorname{dim} G_{r, n}=r(n-r)$ and it is well known that $G_{r, n}$ can be embedded via Plücker morphism in the projective space $\mathbb{P}^{N}=\operatorname{Proj}\left(K\left[x_{0}, \ldots, x_{N}\right]\right)$ with $N=\left(\begin{array}{l}n \\ r\end{array}\right)-1$.

We refer to [6] for generalities about the Grassmannian variety.

We recall also this well known result that we use in the following. We give a proof for the convenience of the reader.

Lemma 2.1. Let $L$ be a line contained in $G$. Then the normal sheaf of $L$ restricted to $G$ is

$$
\mathcal{N}_{L \mid G} \cong \mathcal{O}_{L}^{n-2}(1) \oplus \mathcal{O}_{L}^{(r-1)(n-r-1)}
$$

Proof. Let $Q$ ( $S$, respectively) be the tautological quotient bundle of rank $r$ ( tautological quotient subbundle of rank $n-r$, respect.) of the Grassmannian $G=G_{r, n}$. We have that the tangent bundles of the Grassmannian variety $G$ and of the line $L$ are $\mathcal{T} G \cong Q \otimes S^{*}$ (see [6], p. 201) and $\mathcal{T} L \cong \mathcal{O}_{L}(2)$. Moreover, $Q_{\mid L} \cong \mathcal{O}_{L}(1) \oplus \mathcal{O}_{L}^{r-1}$ and $S_{\mid L}^{*} \cong \mathcal{O}_{L}(1) \oplus \mathcal{O}_{L}^{n-r-1}$ because $Q$ and $S^{*}$ are spanned, $\operatorname{det}(Q) \cong \mathcal{O}_{G}(1)$, and $\operatorname{det}\left(S^{*}\right) \cong \mathcal{O}_{G}(1)$. Then, we get $\mathcal{T} G_{\mid L} \cong \mathcal{O}_{L}(2) \oplus \mathcal{O}_{L}(1)^{n-2} \oplus \mathcal{O}_{L}^{(r-1)(n-r-1)}$ and from the exact sequence:

$$
0 \rightarrow \mathcal{T} L \rightarrow \mathcal{T} G_{\mid L} \rightarrow \mathcal{N}_{L \mid G} \rightarrow 0
$$

we can compute $\mathcal{N}_{L \mid G}$.

Remark 2.2. The isomorphism $\mathcal{N}_{L \mid G} \cong \mathcal{O}_{L}^{n-2}(1) \oplus \mathcal{O}_{L}^{(r-1)(n-r-1)}$ is the key point of our construction and for this reason the construction holds also for every rational smooth variety $U$ containing $L$ such that $\mathcal{N}_{L \mid U}=\mathcal{O}_{L}^{s}(1) \oplus \mathcal{O}_{L}^{t}$, with $s \geq 1$ and $t \geq 0$.

Now, we recall the definition of a $d$-rope following [5]. This definition applies for a rope $C$ not necessarily embedded in a projective space (and supported on an irreducible smooth curve).

Definition 2.3. A $d$-rope $C$ is a projective scheme such that:

(i) $L=C_{\text {red }}$ is an irreducible smooth curve;

(ii) the ideal sheaf $\mathcal{I}=\mathcal{I}_{L, C}$ has $\mathcal{I}^{2}=0$ and hence is an $\mathcal{O}_{L}$-module;

(iii) $\mathcal{I}$ is locally free of rank $d-1$ over $L$.

In the following the scheme $L$ will be a line.

As recalled in Section 1, it is easy to see that a $d$-rope supported on a line $L$ corresponds to a rank $d-1$ subbundle $E$ of the normal bundle $\mathcal{N}_{L}$ of $L$ via the exact sequence $(1)$

$$
0 \rightarrow E^{*}=\mathcal{I}_{L, C} \longrightarrow \mathcal{O}_{C} \longrightarrow \mathcal{O}_{L} \rightarrow 0 \text {. }
$$

The subbundle $E^{*}$ is the conormal bundle of $L$ in $C$. 
Theorem 2.4. A d-rope $C$ supported on a line $L \subset G_{r, n}$ defined by

$$
E^{*}=\oplus_{i=1}^{d-1} \mathcal{O}_{L}\left(\alpha_{i}-1\right)
$$

can be embedded into $G_{r, n}$ if, and only if, either

(i) $d<\operatorname{dim} G_{r, n}$;

(ii) $\alpha_{i} \geq 0 \forall i$;

(iii) $n \geq 2+\left|\left\{i \mid \alpha_{i}=0\right\}\right|$;

or

(i) $d=\operatorname{dim} G_{r, n}$;

(ii) $\alpha_{i}=0,1 \forall i$;

(iii) $\left|\left\{i \mid \alpha_{i}=0\right\}\right|=(r-1)(n-r-1)$, and $\left|\left\{i \mid \alpha_{i}=1\right\}\right|=n-2$.

Proof. According to Definition 2.3 and recalling that $G_{r, n}$ is smooth in a neighborhood of $L$, the rope $C$ can be embedded into $G_{r, n}$ by $\mathcal{O}_{C}(1)$ if, and only if, we can give an injective map

$$
E=\oplus_{i=1}^{d-1} \mathcal{O}_{L}\left(1-\alpha_{i}\right) \rightarrow \mathcal{N}_{L \mid G}=\mathcal{O}_{L}^{n-2}(1) \oplus \mathcal{O}_{L}^{(r-1)(n-r-1)}
$$

which does not drop rank in codimension 1 , that is to say, it provides an embedding of vector bundles.

This is possible if, and only if, $\left(1-\alpha_{i}\right) \leq 1, \forall i=1, \ldots, d-1$, and either $r k(E)<$ $r k\left(\mathcal{N}_{L \mid G}\right)$ i. e. $\operatorname{dim} G>d$, and at most $n-2$ integers $\alpha_{i}-1$ are equal to -1 , or $r k(E)=r k\left(\mathcal{N}_{L \mid G}\right)$, i. e. $\operatorname{dim} G=d$, and $E \cong \mathcal{N}_{L \mid G}$.

From the embedding of vector bundles (3), the exact sequence (2), and the fact that $E$ is a free $\mathcal{O}_{L}$-module, we get that there exists a surjective morphism $\mathcal{T} G_{\mid L} \rightarrow E$ whose kernel is $\mathcal{T} \hat{E} C$, and so the claim follows.

Remark 2.5. For every $x \in L=C_{\text {red }}, \mathcal{T} C_{x}$ has dimension $\operatorname{dim} G-d+1$.

Corollary 2.6. If $C$ is a d-rope embedded into a Grassmannian variety $G$ and supported on a line $L \subset G$ then $\mathcal{O}_{C}$ is an $\mathcal{O}_{L}$-module and the sequence (1) splits as sequence of $\mathcal{O}_{L}$-modules.

Proof. If we apply $\operatorname{Hom}\left(\mathcal{O}_{L},-\right)$ to the sequence (1), we get

$$
\cdots \rightarrow \operatorname{Hom}\left(\mathcal{O}_{L}, \mathcal{O}_{C}\right) \rightarrow \operatorname{Hom}\left(\mathcal{O}_{L}, \mathcal{O}_{L}\right) \rightarrow \operatorname{Ext}^{1}\left(\mathcal{O}_{L}, E^{*}\right) \rightarrow \cdots .
$$

By [7], Ch. III, Proposition 6.3(c), $\operatorname{Ext}^{1}\left(\mathcal{O}_{L}, E^{*}\right) \cong H^{1}\left(L, E^{*}\right)=0$ because of previous Theorem 2.4, and so we have the surjectivity of the map $\operatorname{Hom}\left(\mathcal{O}_{L}, \mathcal{O}_{C}\right) \rightarrow$ $\operatorname{Hom}\left(\mathcal{O}_{L}, \mathcal{O}_{L}\right)$. Hence, there exists a map $\psi: \mathcal{O}_{L} \rightarrow \mathcal{O}_{C}$ which lifts the identity $i d_{L}: \mathcal{O}_{L} \rightarrow \mathcal{O}_{L}$, that is to say, $\mathcal{O}_{C}$ is an $\mathcal{O}_{L}$-module and the sequence (1) splits as sequence of $\mathcal{O}_{L}$-modules. 
Remark 2.7. (i) Previous Corollary 2.6 proves that the split ropes supported on a line, which are the simplest possible abstract ropes, are the only one that can be embedded in a Grassmannian variety.

(ii) The existence of a retraction of the map $\mathcal{O}_{C} \rightarrow \mathcal{O}_{L}$ of the sequence (1) can be proved directly using a projection argument, as in [3], Lemma 2.6

Remark 2.8. If the conditions of Theorem 2.4 hold, then the map

$$
E \rightarrow \mathcal{N}_{L \mid G}
$$

induces a surjective map:

$$
\mathcal{N}_{L \mid G}^{*} \rightarrow E^{*}
$$

and so we can deduce the exact sequence:

$$
0 \rightarrow \mathcal{K} \rightarrow \mathcal{N}_{L \mid G}^{*} \rightarrow E^{*} \rightarrow 0
$$

which can be also written as

$$
\begin{aligned}
0 \rightarrow \oplus_{j=1}^{\operatorname{dim} G-d} \mathcal{O}_{L}\left(-\beta_{j}-1\right) & \stackrel{\varphi_{B}}{\longrightarrow} \\
& \stackrel{\varphi_{B}}{\longrightarrow} \mathcal{O}_{L}^{n-2}(-1) \oplus \mathcal{O}_{L}^{(r-1)(n-r-1)} \stackrel{\varphi_{A}}{\longrightarrow} \oplus_{i=1}^{d-1} \mathcal{O}_{L}\left(\alpha_{i}-1\right) \rightarrow 0
\end{aligned}
$$

Now, we can deduce some numerical invariants for the rope $C$ embedded in $G_{r, n}$.

Proposition 2.9. Let $C$ be a d-rope supported on a line and embedded in the Grassmannian variety $G=G_{r, n}$. We have:

(i) $\sum_{j=1}^{\operatorname{dim} G-d} \beta_{j}=\sum_{i=1}^{d-1} \alpha_{i}+n-1-\operatorname{dim} G$;

(ii) $g_{C}=-\sum_{i=1}^{d-1} \alpha_{i}$.

Proof. (i) Computing the Hilbert polynomials from (4), we get:

$$
\sum_{j=1}^{\operatorname{dim} G-d}\left(\begin{array}{c}
z-\beta_{j} \\
1
\end{array}\right)-(n-2)\left(\begin{array}{l}
z \\
1
\end{array}\right)-(r-1)(n-r-1)\left(\begin{array}{c}
z+1 \\
1
\end{array}\right)+\sum_{i=1}^{d-1}\left(\begin{array}{c}
z+\alpha_{i} \\
1
\end{array}\right)=0 .
$$

Now, an easy computation gives the first claim.

(ii) We have that $\chi\left(\mathcal{O}_{L}(z)\right)=z+1$ and $\chi\left(E^{*}(z)\right)=\sum_{i=1}^{d-1}\left(z+\alpha_{i}\right)=(d-1) z+$ $\sum_{i=1}^{d-1} \alpha_{i}$. Then, we obtain $\chi\left(\mathcal{O}_{C}(z)\right)=d z+1+\sum_{i=1}^{d-1} \alpha_{i}$ which gives the genus.

Remark 2.10. In [10] the authors studied $d$-ropes supported on a line, embedded in $\mathbb{P}^{n}$. In particular they consider the exact sequence

$$
0 \rightarrow \oplus_{j=1}^{n-d} \mathcal{O}_{\mathbb{P}^{1}}\left(-\beta_{j}-1\right) \stackrel{\varphi_{B}}{\longrightarrow} \mathcal{O}_{\mathbb{P}^{1}}^{n-1}(-1) \stackrel{\varphi_{A}}{\longrightarrow} \oplus_{i=1}^{d-1} \mathcal{O}_{\mathbb{P}^{1}}\left(\alpha_{i}-1\right) \rightarrow 0
$$


similar to the sequence (4), which defines a $d$-rope $C$ in $\mathbb{P}^{n}$. Using this sequence, they deduce that $\sum_{j=1}^{n-d} \beta_{j}=\sum_{i=1}^{d-1} \alpha_{i}$ and $g_{C}=-\sum_{i=1}^{d-1} \alpha_{i}$ (see Lemma 2.8 and Proposition 2.9 in $[10])$.

We observe that both for ropes in $\mathbb{P}^{n}$ and for ropes in $G_{r, n}$ the genus depends on the twist of the sheaf $E$. Moreover, the relations between the shifts $\alpha_{i}$ and $\beta_{j}$ are different and depend on the Grassmannian variety where the rope is embedded.

\section{Vector bundles on ropes on the Grassmannian variety}

In this section we want to describe the vector bundles $A$ on a $d$-rope $C$ supported on a line $L$, both embedded in $G_{r, n}$, under some constrains on $C$.

In the previous section we characterized a $d$-rope $C$, supported on $L$ and embedded in $G$, using the sheaf $E^{*}=\oplus_{i=1}^{d-1} \mathcal{O}_{L}\left(\alpha_{i}-1\right)$.

We need the following definitions

Definition 3.1. We say that $C$ is semipositive if $\alpha_{i} \geq 1, \forall i=1, \ldots, d-1$.

Definition 3.2. We say that a sheaf $\oplus_{i=1}^{m} \mathcal{O}_{L}\left(a_{i}\right)$, with $a_{1} \geq a_{2} \geq \cdots \geq a_{m}$, is rigid if $a_{m} \geq a_{1}-1$.

For the semipositive ropes on $G$ we have:

Proposition 3.3. Let $A$ be a vector bundle on a semipositive d-rope $C$ supported on a line $L$ embedded in a Grassmannian variety $G$. If $A_{\mid L} \cong \oplus_{i \in I} \mathcal{O}_{L}\left(a_{i}\right)$ is rigid then $A \cong \oplus_{i \in I} \mathcal{O}_{C}\left(a_{i}\right)$.

Proof. We set $B=\oplus_{i \in I} \mathcal{O}_{C}\left(a_{i}\right)$. We have that $B_{\mid L} \cong A_{\mid L}$ or, more precisely, there is an isomorphism:

$$
\psi: A_{\mid L} \rightarrow B_{\mid L} .
$$

We have that $\mathcal{H o m}(A, B)_{\mid L} \cong \mathcal{H} o m\left(\oplus_{i \in I} \mathcal{O}_{L}\left(a_{i}\right), \oplus_{j \in I} \mathcal{O}_{L}\left(a_{j}\right)\right) \cong \oplus_{i, j \in I} \mathcal{O}_{L}\left(a_{j}-a_{i}\right)$ and for the rigidity, we have that $-1 \leq a_{j}-a_{i} \leq 1$.

If we tensorize the exact sequence (1)

$$
0 \rightarrow E^{*} \rightarrow \mathcal{O}_{C} \rightarrow \mathcal{O}_{L} \rightarrow 0
$$

by $\mathcal{H o m}(A, B)$ and if we write the associated cohomology sequence, we obtain:

$$
\begin{aligned}
0 \rightarrow H^{0}\left(E^{*} \otimes_{\mathcal{O}_{L}}\right. & \mathcal{H o m}(A, B)) \rightarrow H^{0}\left(\mathcal{O}_{C} \otimes_{\mathcal{O}_{L}} \mathcal{H o m}(A, B)\right) \rightarrow \\
& \rightarrow H^{0}\left(\mathcal{O}_{L} \otimes_{\mathcal{O}_{L}} \mathcal{H o m}(A, B)\right) \rightarrow H^{1}\left(E^{*} \otimes_{\mathcal{O}_{L}} \mathcal{H o m}(A, B)\right) \rightarrow \cdots
\end{aligned}
$$

In fact $E^{*} \otimes_{\mathcal{O}_{L}} \mathcal{H o m}(A, B) \cong \oplus_{i=1}^{m} \mathcal{O}_{L}\left(\alpha_{i}-1\right) \otimes_{\mathcal{O}_{L}} \mathcal{H o m}(A, B)$ and this is a sum of line bundles twisted by $\omega_{i} \geq-1$.

Then, the isomorphism $\psi \in H^{0}\left(\mathcal{O}_{L} \otimes_{\mathcal{O}_{L}} \mathcal{H o m}(A, B)\right)$ can be lifted to a morphism $\psi^{\prime} \in H^{0}\left(\mathcal{O}_{C} \otimes_{\mathcal{O}_{L}} \mathcal{H}\right.$ om $\left.(A, B)\right)$. By Nakayama's Lemma the morphism $\psi^{\prime}$ is an isomorphism, too. 
Remark 3.4. Let $C$ be a semipositive $d$-rope supported on a line $L$ both embedded in a smooth rational variety $U$ (see also Remark 2.2) with $\mathcal{O}_{U}(1)_{\mid L}=\mathcal{O}_{L}(1)$. If $A$ is a vector bundle on $C$ such that $A_{\mid L} \cong \mathcal{O}_{L}(a)^{m}$, then $A \cong \mathcal{O}_{C}(a)^{m}$. In fact, the same proof as in Proposition 3.3 works in this case too, pointing out that $\mathcal{H o m}(A, B) \cong \mathcal{O}_{L}^{r^{2}}$.

\section{A lifting problem}

In this section we want to show that a $d$-rope $C$ supported on a line $L$, both embedded in the Grassmannian $G=G_{r, n} \subseteq \mathbb{P}^{N}$, with $N=\left(\begin{array}{l}n \\ r\end{array}\right)-1$, can be lifted to a $d^{\prime}$-rope $C^{\prime}$ of $\mathbb{P}^{N}$ such that $C$ is the scheme-theoretical intersection of $C^{\prime}$ and $G$.

Lemma 4.1. Let $\mathcal{N}_{G, \mathbb{P}^{N}}$ be the normal sheaf of the Grassmannian $G$ embedded via Plücher morphism in $\mathbb{P}^{N}$. Then

$$
\left(\mathcal{N}_{G, \mathbb{P}^{N}}\right)_{\mid L} \cong \mathcal{O}_{L}^{m_{1}}(1) \oplus \mathcal{O}_{L}^{m_{2}}(2)
$$

where $m_{1}=N-2 \operatorname{dim} G+n-1$ and $m_{2}=\operatorname{dim} G-n+1$.

Proof. The Grassmannian variety $G$ is scheme-theoretically cut out by quadrics in $\mathbb{P}^{N}$. Then $\mathcal{I}_{G, \mathbb{P}^{N}}(2)$ is spanned and so

$$
\left[\left(\frac{\mathcal{I}_{G, \mathbb{P}^{N}}}{\mathcal{I}_{G, \mathbb{P}^{N}}^{2}}\right)(2)\right]_{\mid L} \cong\left(\mathcal{N}_{G, \mathbb{P}^{N}}^{*}(2)\right)_{\mid L} \cong \oplus \mathcal{O}_{L}\left(a_{i}\right)
$$

with $a_{i} \geq 0, \forall i$, i. e. $\left(\mathcal{N}_{G, \mathbb{P}^{N}}\right)_{\mid L} \cong \oplus \mathcal{O}_{L}\left(b_{i}\right)$, with $b_{i} \leq 2$. Let us consider the exact sequence:

$$
0 \rightarrow \mathcal{N}_{L, G} \rightarrow \mathcal{N}_{L, \mathbb{P}^{N}} \rightarrow\left(\mathcal{N}_{G, \mathbb{P}^{N}}\right)_{\mid L} \rightarrow 0
$$

where $\mathcal{N}_{L, G} \cong \mathcal{O}_{L}^{p} \oplus \mathcal{O}_{L}^{q}(1)$ with $p=(r-1)(n-r-1), q=n-2$ and $\mathcal{N}_{L, \mathbb{P} N} \cong \mathcal{O}_{L}^{N-1}(1)$.

We deduce that $\left(\mathcal{N}_{G, \mathbb{P}^{N}}\right)_{\mid L}$ is ample and then $b_{i} \geq 1$ and $\left(\mathcal{N}_{G, \mathbb{P}^{N}}\right)_{\mid L} \cong \mathcal{O}_{L}^{m_{1}}(1) \oplus$ $\mathcal{O}_{L}^{m_{2}}(2)$ with $m_{1}+m_{2}=N-\operatorname{dim} G$, by rank argument. Moreover, comparing the first Chern classes, we get $m_{1}+2 m_{2}=N-n+1$. A simply calculation gives the claim.

Remark 4.2. We can prove the same result if we consider a scheme $U \subset \mathbb{P}^{N}$ with $L \subset U$ such that $\mathcal{N}_{L, U} \cong \mathcal{O}_{L}^{\alpha} \oplus \mathcal{O}_{L}^{\beta}(1)$. In this case we have that $\left(\mathcal{N}_{U, \mathbb{P}^{N}}\right)_{\mid L} \cong \mathcal{O}_{L}\left(c_{i}\right)$.

For example we can take any homogeneous smooth variety as $U$.

Now, we consider $L \subset G \subset \mathbb{P}^{N}$. In Section 2 we showed that we can construct a rope supported on $L$ contained in $G$, using the exact sequence (4):

$0 \rightarrow \oplus_{j=1}^{\operatorname{dim} G-d} \mathcal{O}_{L}\left(-\beta_{j}-1\right) \stackrel{\varphi_{B}}{\longrightarrow} \mathcal{O}_{L}^{n-2}(-1) \oplus \mathcal{O}_{L}^{(r-1)(n-r-1)} \stackrel{\varphi_{A}}{\longrightarrow} \oplus_{i=1}^{d-1} \mathcal{O}_{L}\left(\alpha_{i}-1\right) \rightarrow 0$

We can also consider the dualized exact sequence of normal sheaves written in the proof of Lemma 4.1:

$$
0 \rightarrow\left(\mathcal{N}_{G, \mathbb{P}^{N}}^{*}\right)_{\mid L} \rightarrow \mathcal{N}_{L, \mathbb{P}^{N}}^{*} \rightarrow \mathcal{N}_{L, G}^{*} \rightarrow 0
$$


Pointing out that $\mathcal{N}_{L, G}^{*} \cong \mathcal{O}_{L}^{n-2}(-1) \oplus \mathcal{O}_{L}^{(r-1)(n-r-1)}$, we construct the following diagram:

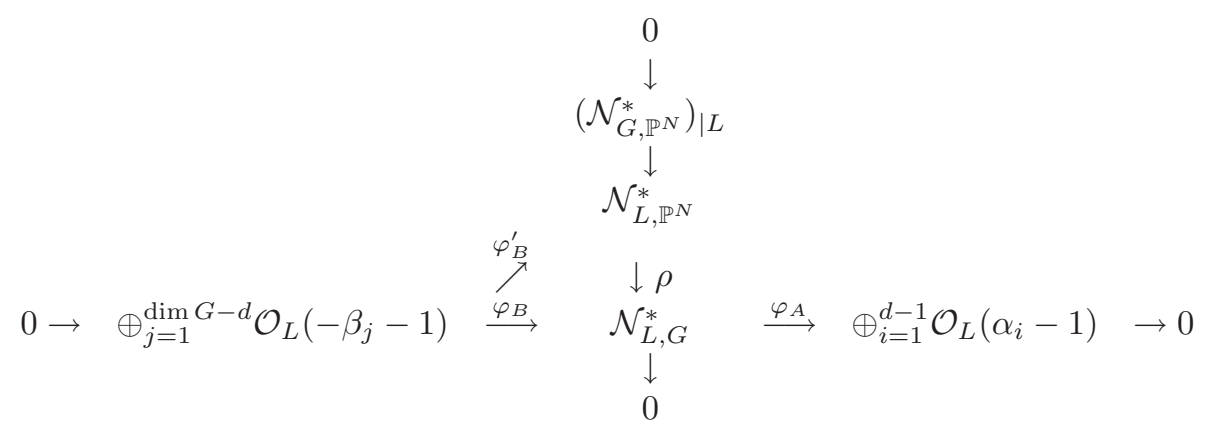

Theorem 4.3. With the notation as above, suppose that $\beta_{j} \geq 0$ for all $j$. We can lift the morphism $\varphi_{B}$ to a morphism $\varphi_{B}^{\prime}: \oplus_{j=1}^{\operatorname{dim} G-d} \mathcal{O}_{L}\left(-\beta_{j}-1\right) \rightarrow \mathcal{N}_{L, \mathbb{P} N}^{*}$ (not uniquely) which gives a $d^{\prime}$-rope $C^{\prime} \subset \mathbb{P}^{N}$, supported on $L$, with $d^{\prime}=N-\operatorname{dim} G+d$.

We have that $C$ is the scheme-theoretical intersection of $C^{\prime}$ with $G$.

Proof. Applying $\operatorname{Hom}\left(\oplus_{j} \mathcal{O}_{L}\left(-\beta_{j}-1\right),-\right)$ to the sequence (5) we get:

$$
\begin{aligned}
0 \rightarrow \operatorname{Hom} & \left(\oplus_{j} \mathcal{O}_{L}\left(-\beta_{j}-1\right),\left(\mathcal{N}_{G, \mathbb{P}^{N}}^{*}\right)_{\mid L}\right) \rightarrow \\
& \rightarrow \operatorname{Hom}\left(\oplus_{j} \mathcal{O}_{L}\left(-\beta_{j}-1\right),\left(\mathcal{N}_{L, \mathbb{P}^{N}}^{*}\right)_{\mid L}\right) \rightarrow \operatorname{Hom}\left(\oplus_{j} \mathcal{O}_{L}\left(-\beta_{j}-1\right), \mathcal{N}_{L, G}^{*}\right) \rightarrow 0 .
\end{aligned}
$$

If we write the associated cohomology sequence we have that

$$
H^{1}\left(\mathcal{H o m}\left(\oplus_{j} \mathcal{O}_{L}\left(-\beta_{j}-1\right), \mathcal{N}_{G, \mathbb{P}^{N} \mid L}^{*}\right)\right)=0
$$

because $\beta_{j} \geq 0$ and then the morphism $\varphi_{B} \in \operatorname{Hom}\left(\oplus_{j} \mathcal{O}_{L}\left(-\beta_{j}-1\right), \mathcal{N}_{L, G}^{*}\right)$ can be lifted (not uniquely) to a morphism $\varphi_{B}^{\prime} \in \operatorname{Hom}\left(\oplus_{j} \mathcal{O}_{L}\left(-\beta_{j}-1\right), \mathcal{N}_{L, \mathbb{P} N}^{*}\right)$ which gives the $d^{\prime}$ rope $C^{\prime}$. The commutativity of the diagram $\left(\rho \varphi_{B}^{\prime}=\varphi_{B}\right)$ assures that $C=C^{\prime} \cap G$, while $d^{\prime}$ can be computed as $N-r k\left(\varphi_{B}^{\prime}\right)=N-\operatorname{dim} G+d$ (cf. [10], Remark 2.5 (i)).

Remarks 4.4. (i) (Geometrical meaning) In some sense, given a rope $C$ on $G$ we can fat the directions transverse to $G$ obtaining a rope in $\mathbb{P}^{N}$.

(ii) We can prove the proposition replacing $G$ with a scheme $U$ satisfying the conditions introduced in Remark 4.2 and with the extra assumption $\beta_{j} \geq c-1$ where $c=\max _{j}\left\{c_{j}\right\}$.

(iii) The lifted ropes $C^{\prime}$ are completely studied in [10]. 


\section{Families of ropes on the Grassmannian variety}

Whenever we want to construct a rope $C$ on $G$ we start with a sheaf

$$
E^{*}=\oplus_{i=1}^{d-1} \mathcal{O}_{L}\left(\alpha_{i}-1\right)
$$

with $\alpha_{i} \geq 0$ and we fix a surjective morphism

$$
\varphi_{A}: \mathcal{O}_{L}^{n-2}(-1) \oplus \mathcal{O}_{L}^{(r-1)(n-r-1)} \rightarrow \oplus_{i=1}^{d-1} \mathcal{O}_{L}\left(\alpha_{i}-1\right)
$$

As shown in Section 2 we naturally get the sequence (4):

$0 \rightarrow \oplus_{j=1}^{\operatorname{dim}} G-d \mathcal{O}_{L}\left(-\beta_{j}-1\right) \stackrel{\varphi_{B}}{\longrightarrow} \mathcal{O}_{L}^{n-2}(-1) \oplus \mathcal{O}_{L}^{(r-1)(n-r-1)} \stackrel{\varphi_{A}}{\longrightarrow} \oplus_{i=1}^{d-1} \mathcal{O}_{L}\left(\alpha_{i}-1\right) \rightarrow 0$

To fix notation, suppose that in (4) $\alpha_{1} \geq \alpha_{2} \geq \cdots \geq \alpha_{d-1}$ and $\beta_{\operatorname{dim} G-d} \geq \cdots \geq$ $\beta_{2} \geq \beta_{1}>0$.

The decreasing sequence of integers $\underline{\alpha}=\left(\alpha_{1}, \ldots, \alpha_{d-1}\right)$ is the splitting type of $E^{*}(1)$ and analogously, the decreasing sequence of integers $\underline{\beta}=\left(-\beta_{1}, \ldots,-\beta_{\operatorname{dim} G-d}\right)$ is the splitting type of $\operatorname{ker} \varphi_{A}(1)$.

Definition 5.1. We say that the sequence of integers $\underline{\alpha}=\left(\alpha_{1}, \ldots, \alpha_{d-1}\right)$ is admissible if $E^{*}=\oplus_{i=1}^{d-1} \mathcal{O}_{L}\left(\alpha_{i}-1\right)$ satisfies the hypotheses of Theorem 2.4.

The sequence of integers $\beta=\left(-\beta_{1}, \ldots,-\beta_{\operatorname{dim} G-d}\right)$ is admissible if there exists an admissible sequence $\underline{\alpha}$ such that in the exact sequence (4) $\operatorname{ker} \varphi_{A}$ has splitting type $\beta$.

The pair $(\underline{\alpha}, \underline{\beta})$ is admissible if $\underline{\alpha}$ is admissible and $\operatorname{ker} \varphi_{A}$ has splitting type $\underline{\beta}$.

If the rope $C$ on $G$ is associated to the sequence (4) we say that $C$ has $\alpha$-type $\underline{\alpha}$ and $\beta$-type $\beta$.

We define the degree of $\underline{\alpha}(\beta$ respect. $) \quad$ as $\operatorname{deg} \underline{\alpha}=\sum_{i} \alpha_{i}\left(\operatorname{deg} \beta=-\sum_{i} \beta_{i}\right.$, respect.). Now, we define the following partial order between splitting types of the same degree.

Definition 5.2. Let $\underline{\alpha}_{1}=\left(\alpha_{1,1}, \ldots, \alpha_{1, d-1}\right)$ and $\underline{\alpha}_{2}=\left(\alpha_{2,1}, \ldots, \alpha_{2, d-1}\right)$ be two $\alpha$ types of the same degree. We put: $\underline{\alpha}_{1} \geq \underline{\alpha}_{2}$ if $\alpha_{1,1}+\cdots+\alpha_{1, j} \leq \alpha_{2,1}+\cdots+\alpha_{2, j}$ for $1 \leq j \leq d-1$.

The analogous definition holds for the $\beta$-types.

We can also define a partial order between the admissible pairs.

Definition 5.3. Let $\left(\underline{\alpha}_{1}, \underline{\beta}_{1}\right)$ and $\left(\underline{\alpha}_{2}, \underline{\beta}_{2}\right)$ be two admissible pairs. We say that $\left(\underline{\alpha}_{1}, \underline{\beta}_{1}\right) \geq\left(\underline{\alpha}_{2}, \underline{\beta}_{2}\right)$ if $\underline{\alpha}_{1} \geq \underline{\alpha}_{2}$ and $\underline{\beta}_{1} \geq \underline{\beta}_{2}^{2}$ according with Definition 5.2.

Now, let $\Gamma_{\underline{\alpha}}$ be the set of all ropes $C$ with admissible $\alpha$-type $\underline{\alpha}, \Delta_{\beta}$ the set of all ropes $C$ with admissible $\beta$-type $\underline{\beta}$ and let $\Omega_{(\underline{\alpha}, \beta)}$ be the non-empty set of all ropes $C$ with $\alpha$-type $\underline{\alpha}$ and $\beta$-type $\beta$, with $(\underline{\alpha}, \beta)$ admissible pair.

For ropes $C$ in $G_{r, n}$ we can state a result analogous to Theorem 1 in [2] and we can prove it with similar arguments. 
Theorem 5.4. Let $\left(\underline{\alpha}_{1}, \underline{\beta}_{1}\right)$ and $\left(\underline{\alpha}_{2}, \underline{\beta}_{2}\right)$, be two admissible pairs with $\operatorname{deg} \underline{\alpha}_{1}=$ $\operatorname{deg} \underline{\alpha}_{2}, \operatorname{deg} \beta_{1}=\operatorname{deg} \underline{\beta}_{2},\left(\underline{\alpha}_{1}, \beta_{1}\right) \geq\left(\underline{\alpha}_{2}, \underline{\beta}_{2}\right)$ and with the extra assumption $\alpha_{1, d-1} \geq 1$ and $\alpha_{2, d-1} \geq 1$. Let $C \in \Omega_{\left(\underline{\alpha}_{2}, \beta_{2}\right)}$. Then there exists a flat family of ropes in $G$ parameterized by a non empty open subset of an affine line whose special member is $C$ and whose general member is an element of $\Omega_{\left(\underline{\alpha}_{1}, \beta_{1}\right)}$.

We need some preliminary results.

Lemma 5.5. Let $\underline{\alpha}_{1}$ and $\underline{\alpha}_{2}$ be two admissible $\alpha$-types with $\operatorname{deg} \underline{\alpha}_{1}=\operatorname{deg} \underline{\alpha}_{2}$ and $\underline{\alpha}_{1} \geq \underline{\alpha}_{2}$. Let $C \in \bar{\Gamma}_{\underline{\alpha}_{2}}$. Then there exists a flat family of ropes in $G$, (parameterized by a non-empty open subset of an affine line) whose special member is $C$ and whose general member is an element of $\Gamma_{\underline{\alpha}_{1}}$.

Proof. We set $\underline{\alpha}_{1}=\left(\alpha_{1,1}, \ldots, \alpha_{1, d-1}\right)$ and $\underline{\alpha}_{2}=\left(\alpha_{2,1}, \ldots, \alpha_{2, d-1}\right)$. We observe that $\alpha_{1, i} \geq 0, \alpha_{2, i} \geq 0$ for all $i=1, \ldots, d-1$ for the admissibility of the $\alpha$-types.

Because of the inequality $\underline{\alpha}_{1} \geq \underline{\alpha}_{2}$ it is well known that there exists a flat family of rank $d-1$ vector bundles on $L$ (the support of the rope $C$ ) parameterized by an open subset $T$ of an affine line $\mathbb{A}^{1}$ whose special member is $A_{2} \cong \oplus_{i=1}^{d-1} \mathcal{O}_{L}\left(\alpha_{2, i}-1\right)$ and whose general member is isomorphic to $A_{1} \cong \oplus_{i=1}^{d-1} \mathcal{O}_{L}\left(\alpha_{1, i}-1\right)$.

A surjective morphism $f \in H^{0}\left(L, \mathcal{H o m}\left(\mathcal{O}_{L}^{n-2}(-1) \oplus \mathcal{O}_{L}^{(r-1)(n-r-1)}, A_{2}\right)\right)$ induces a rope $C$ in $G$ given by the sequence:

$$
0 \rightarrow \oplus_{j=1}^{\operatorname{dim} G-d} \mathcal{O}_{L}\left(-\beta_{j}-1\right) \stackrel{\varphi_{B}}{\longrightarrow} \mathcal{O}_{L}^{n-2}(-1) \oplus \mathcal{O}_{L}^{(r-1)(n-r-1)} \stackrel{f}{\longrightarrow} A_{2} \rightarrow 0 .
$$

Since $\operatorname{deg} \underline{\alpha}_{1}=\operatorname{deg} \underline{\alpha}_{2}$, we have that

$$
\begin{aligned}
& h^{0}\left(L, \mathcal{H o m}\left(\mathcal{O}_{L}^{n-2}(-1) \oplus \mathcal{O}_{L}^{(r-1)(n-r-1)}, A_{2}\right)\right)= \\
& h^{0}\left(L, \mathcal{H o m}\left(\mathcal{O}_{L}^{n-2}(-1) \oplus \mathcal{O}_{L}^{(r-1)(n-r-1)}, A_{1}\right)\right) .
\end{aligned}
$$

Applying [8], it is easy to check the existence of a vector bundle $E$ on $T$ with $r k(E)=$ $h^{0}\left(L, \mathcal{H o m}\left(\mathcal{O}_{L}^{n-2}(-1) \oplus \mathcal{O}_{L}^{(r-1)(n-r-1)}, A_{1}\right)\right)$ which is a universal parameter space for the family of the homomorphisms parameterized by $T$, that is to say, $\forall P \in T$ the fibre $E_{P} \cong H^{0}\left(L, \mathcal{H o m}\left(\mathcal{O}_{L}^{n-2}(-1) \oplus \mathcal{O}_{L}^{(r-1)(n-r-1)}, A_{1}\right)\right)$.

Hence, we can find a rational path in the total space $E$ joining the element representing $f$ to a surjection $g: \mathcal{O}_{L}^{n-2}(-1) \oplus \mathcal{O}_{L}^{(r-1)(n-r-1)} \rightarrow A_{1}$, and so the claim holds.

We can state the same result for the $\underline{\beta}$-types.

Lemma 5.6. Let $\underline{\beta}_{1}$ and $\underline{\beta}_{2}$ be two admissible $\beta$-types for with $\operatorname{deg} \underline{\beta}_{1}=\operatorname{deg} \underline{\beta}_{2}$ and $\underline{\beta}_{1} \geq \underline{\beta}_{2}$. Let us take a rope $C \in \Delta_{\underline{\beta}_{2}}$. Then there exists a flat family of ropes in $G$, (parameterized by a non-empty open subset of an affine line) whose special member is $C$ and whose general member is an element of $\Delta_{\underline{\beta}_{1}}$. 
Proof of Theorem 5.4. Set

$$
A_{1}=\oplus_{i=1}^{d-1} \mathcal{O}_{L}\left(\alpha_{1, i}-1\right), \quad A_{2}=\oplus_{i=1}^{d-1} \mathcal{O}_{L}\left(\alpha_{2, i}-1\right)
$$

and

$$
B_{1}=\oplus_{i=1}^{d-1} \mathcal{O}_{L}\left(-\beta_{1, i}-1\right), \quad B_{2}=\oplus_{i=1}^{d-1} \mathcal{O}_{L}\left(-\beta_{2, i}-1\right) .
$$

Let $C$ be a rope corresponding to a surjective morphism $f \in H^{0}\left(\mathcal{H o m}\left(\mathcal{O}_{L}^{n-2}(-1) \oplus\right.\right.$ $\left.\left.\mathcal{O}_{L}^{(r-1)(n-r-1)}, A_{2}\right)\right)$. The rope $C$ is defined by an extension of $A_{2}$ by $B_{2}$ with middle term isomorphic to $\mathcal{O}_{L}^{n-2}(-1) \oplus \mathcal{O}_{L}^{(r-1)(n-r-1)}$ (which is a rigid bundle). Since there exists such an extension, by semicontinuity the general extension of $A_{2}$ and $B_{2}$ has middle term isomorphic to $\mathcal{O}_{L}^{n-2}(-1) \oplus \mathcal{O}_{L}^{(r-1)(n-r-1)}$.

As in the proof of Lemma 5.5, we have that there exists a flat family of pairs of vector bundles on $L$, parameterized by an open subset $T$ of the affine line with $\left(A_{2}, B_{2}\right)$ as special fiber and $\left(A_{1}, B_{1}\right)$ as general fibre.

Since the pairs $\left(\underline{\alpha}_{i}, \underline{\beta}_{i}\right)$ are admissible, for $i=1,2$, and $\alpha_{i, d-1} \geq 1$, for $i=1,2$ then $h^{0}\left(L, \mathcal{H o m}\left(A_{1}, B_{1}\right)\right)=h^{0}\left(L, \mathcal{H o m}\left(A_{2}, B_{2}\right)\right)=0$ and so, using Riemann-Roch Theorem we get $h^{1}\left(L, \mathcal{H o m}\left(A_{1}, B_{1}\right)\right)=h^{1}\left(L, \mathcal{H o m}\left(A_{2}, B_{2}\right)\right)$.

This implies that there exists a vector bundle $E$ on $T$ with

$$
r k(E)=h^{1}\left(L, \mathcal{H o m}\left(A_{1}, B_{1}\right)\right)=h^{1}\left(L, \mathcal{H o m}\left(A_{2}, B_{2}\right)\right)
$$

such that $\forall P \in T$ the fibre $E_{P}=\left(\left(A_{1}\right)_{P},\left(B_{1}\right)_{P}\right)$ is isomorphic to $H^{1}\left(L, \mathcal{H o m}\left(\left(A_{1}\right)_{P}\right.\right.$, $\left.\left.\left(B_{1}\right)_{P}\right)\right)$.

By semicontinuity, for every $P \in T$ the general extension of $\left(A_{1}\right)_{P}$ by $\left(B_{1}\right)_{P}$ has middle term isomorphic to $\mathcal{O}_{L}^{n-2}(-1) \oplus \mathcal{O}_{L}^{(r-1)(n-r-1)}$ and so it defines a rope embedded in the Grassmannian $G_{r, n}$ for every $P$. In fact, we have a line $L \subset G_{r, n}$ and an exact sequence as (4), which defines the scheme structure of the rope embedded in $G_{r, n}$. The family of such extensions is algebraic and projective. Furthermore, the degree and genus of the ropes we obtain are fixed because $\operatorname{deg}(C)=\operatorname{rank}_{K}\left(A_{1}\right)_{P}+1=$ $\operatorname{rank}_{K}\left(A_{2}\right)_{P}+1$, and $g(C)=-\operatorname{deg}\left(\underline{\alpha}_{1}\right)=-\operatorname{deg}\left(\underline{\alpha}_{2}\right)$. The Hilbert polynomial of the ropes is then independent of $P$ and so the family is flat by [7], Ch. III, Theorem 9.9.

As last result, we describe a parameter space for the set $\Gamma_{\underline{\alpha}}$. Of course, a similar statement holds for $\Delta_{\underline{\beta}}$.

Proposition 5.7. The scheme structures of ropes in $\Gamma_{\underline{\alpha}}$ are parameterized by a nonempty, irreducible, rational variety $\mathcal{U}$ of dimension

$$
\operatorname{dim} \mathcal{U}=\operatorname{deg} \underline{\alpha}(\operatorname{dim} G-1)+(n-2)(d-1)-\sum_{i, j=1}^{d-1}\left(\begin{array}{c}
\alpha_{i}-\alpha_{j}+1 \\
1
\end{array}\right) .
$$

$\Gamma_{\underline{\alpha}}$ is parameterized by $F_{1}(G) \times \mathcal{U}$, where $F_{1}(G)$ is the Fano variety of the lines in $G$. 
Proof. Let $\underline{\alpha}=\left(\alpha_{1}, \ldots, \alpha_{d-1}\right)$ and let $\mathcal{A}=\oplus_{i=1}^{d-1} \mathcal{O}_{\mathbb{P}^{1}}\left(\alpha_{i}-1\right)$.

Every rope in $\Gamma_{\underline{\alpha}}$ is uniquely determined by a pair $(L, \mathcal{E})$ where $L$ is a line in $G$ and $\mathcal{E}=\operatorname{Im}\left(\varphi_{A}^{*}\right) \subset \mathcal{N}_{L \mid G}$, with $\varphi_{A} \in \operatorname{Hom}\left(\mathcal{N}_{L \mid G}, \mathcal{A}\right)$. Then, the parameter space for the scheme structures of ropes in $\Gamma_{\underline{\alpha}}$ supported on a fixed line is the quotient of the open subset $\mathcal{U}$ of $\operatorname{Hom}\left(\mathcal{N}_{L \mid G}, \mathcal{A}\right)$, corresponding to surjective maps which do not drop rank in codimension 1 , by the action of the automorphisms of $\mathcal{A}$.

Hence, $\mathcal{U}$ is irreducible, rational of dimension

$$
\begin{aligned}
\operatorname{dim} \mathcal{U} & =h^{0}\left(\operatorname{Hom}\left(\mathcal{N}_{L \mid G}, \mathcal{A}\right)\right)-\operatorname{dim} \operatorname{Aut} \mathcal{A}= \\
& =\operatorname{deg}(\underline{\alpha})(\operatorname{dim} G-1)+(d-1)(n-2)-\sum_{i, j=1}^{d-1}\left(\begin{array}{c}
\alpha_{j}-\alpha_{i}+1 \\
1
\end{array}\right) .
\end{aligned}
$$

The last part of the statement is straightforward.

Remark 5.8. We want to compute the dimension of $F_{1}(G)$. Each line $L$ in $G$ is determined by a surjective morphism $\mathcal{O}_{\mathbb{P}^{1}}^{n} \rightarrow \mathcal{O}_{\mathbb{P}^{1}}^{r-1} \oplus \mathcal{O}_{\mathbb{P}^{1}}(1)=\mathcal{V}$ because of the universal property of the Grassmannian $G$, up to the automorphisms of $\mathcal{V}$ and the ones of $\mathbb{P}^{1}$. Hence, the dimension of $F_{1}(G)$ is

$$
\operatorname{dim} F_{1}(G)=h^{0}\left(\mathcal{O}_{\mathbb{P}^{1}}^{n(r-1)} \oplus \mathcal{O}_{\mathbb{P}^{1}}^{n}(1)\right)-\operatorname{dim} \operatorname{Aut} \mathcal{V}-3=\operatorname{dim} G+n-3 .
$$

Of course, the parameter space for $\Gamma_{\underline{\alpha}}$ reflects the properties of $F_{1}(G)$.

Acknowledgements. During the preparation of this paper the second author and the third one profited of stays at the University of Trento and they wish to thank this institution for the warm hospitality.

\section{References}

[1] D. Bayer and D. Eisenbud, Ribbons and their canonical embeddings, Trans. Amer. Math. Soc. 347 (1995), 719-756.

[2] E. Ballico, Multiple structures on lines, Int. Math. J. 2 (2002), 695-701.

[3] E. Ballico and R. Notari, Ropes on linear subspaces of a projective space (preprint).

[4] C. Bănică and O. Forster, Multiplicity structures on space curves, The Lefschetz Centennial Conference, Part I (Mexico City, 1984), Contemp. Math., vol. 58, Amer. Math. Soc., Providence, RI, 1986, pp. 47-64.

[5] K. A. Chandler, Geometry of dots and ropes, Trans. Amer. Math. Soc. 347 (1995), 767-784.

[6] J. Harris, Algebraic geometry, Graduate Texts in Mathematics, vol. 133, Springer-Verlag, New York, 1992, ISBN 0-387-97716-3.

[7] R. Hartshorne, Algebraic geometry, Springer-Verlag, New York, 1977, ISBN 0-387-90244-9.

[8] H. Lange, Universal families of extensions, J. Algebra 83 (1983), 101-112. 
[9] J. C. Migliore, C. Peterson, and Y. Pitteloud, Ropes in projective space, J. Math. Kyoto Univ. 36 (1996), 251-278.

[10] U. Nagel, R. Notari, and M. L. Spreafico, Curves of degree two and ropes on a line: their ideals and even liaison classes, J. Algebra 265 (2003), 772-793.

[11] - The Hilbert scheme of degree two curves and certain ropes (preprint). 